\title{
Search of non-standard strong gravity at nuclear scale using electron spin geodetic precession
}

\author{
Saki Tanaka ${ }^{1 \mathrm{a}}$, Yusuke Nakaya ${ }^{1}$, Reiya Narikawa ${ }^{1}$, Kazufumi Ninomiya ${ }^{1}$, Junichi Onishi ${ }^{1}$, Matthew \\ Pearson $^{2}$, Robert Openshaw ${ }^{2}$, Shuntaro Saiba ${ }^{1}$, Ryosuke Tanuma ${ }^{1}$, Yumi Totsuka ${ }^{1}$ and Jiro Murata ${ }^{1}$ \\ ${ }^{1}$ Department of Physics, Rikkyo University, Nishi-Ikebukuro, Tokyo 171-8501, Japan \\ ${ }^{2}$ TRIUMF, Vancouver, BC V6T 2A3, Canada
}

\begin{abstract}
The MTV-G project was started in 2011 to explore a strong gravitational field at a nuclear scale in an electron double scattering experiment, utilizing an experimental technique of the MTV experiment, which searches a electron's T-Violating transverse polarization in nuclear beta decay at TRIUMF-ISAC. In addition to this new experiment, we have also performed a re-analysis of spectroscopic data of exotic atoms, in a gravitational point of view. From these two studies, we set new constraints on possible new Yukawa interaction at sub-mm scale, as a test of gravitational inverse square law.
\end{abstract}

\section{Introduction}

In 1998, the large extra dimension model called "ADD model" was proposed in order to geometrically resolve the hierarchy problem [1]. This model tries to explain why gravitational force is extremely weaker than other three gauge interactions, i.e., strong, weak and electromagnetic interactions, by introducing additional large spatial dimensions which size, $\Lambda$, is as large as $0.1 \mathrm{~mm}$. According to the ADD model, Newton's inverse square law is predicted to be violated at around the $\Lambda$ scale, and to be modified its potential shape with a stronger power at a short range: $r<<\Lambda$. The present MTV-G experiment started to explore this strong gravitational field, at around nuclear scale where gravity may be amplified by a factor of $10^{30-40}$ from the Newton's inverse square law [2].

Although the gravitational contribution is completely negligible in nuclear phenomena assuming Newton's law, it can be shown that gravity may be getting very big in the large extra dimension model. Indeed, in a $4+d$ dimensional space-time, the gravitational potential becomes

$$
V(r)=\left\{\begin{array}{cc}
-G_{4+d} \frac{M m}{r^{1+d}} & (r<\Lambda) \\
-G \frac{M m}{r} & (r>\Lambda)
\end{array}\right.
$$

using Gauss's law, where $G_{4+d}=G \Lambda^{d}$ is a higher dimensional gravitational constant [1]. If we assume that the number of the large extradimension $d$ is larger than one, the gravitational potential at short distance must be modified to be very strong.

\footnotetext{
${ }^{\mathrm{a}}$ Corresponding author: saki_t @ rikkyo.ac.jp
} 
A current parametrization of non-Newtonian corrections to the inverse square law is given as a Yukawa form : $V_{4+d}(r)=-G \frac{M m}{r}\left(1+\alpha e^{-r / \lambda}\right)$. Status of experimental tests of this new Yukawa term can be expressed in an alpha-lambda parameter space as shown in Figure 1.

On the other hand, according to the Einstein's general relativistic theory, warped space-time is regarded as the origin of gravity. By combing this idea with the ADD model, we can assume an existence of a strongly deformed space-time around nuclei. We are trying to utilize this space-time deformation to detect the strong gravitational field, by means of measuring electron's spin precession in an electron-nuclear scattering, by the geodetic precession effect in a warped space-time.

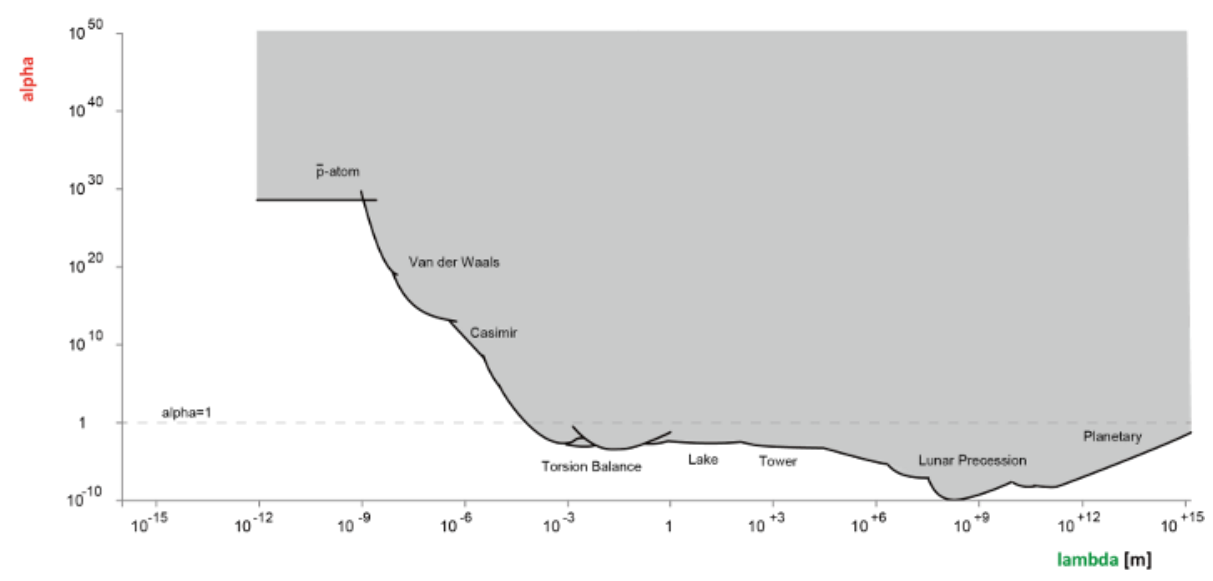

Figure 1. Status of experimental tests of the new Yukawa interaction.

\section{Experiment}

In the present study, electron spin precession is observed in electron-nuclear scattering experiment. The spin polarization vector of the electron emitted from a beta source $\left(37 \mathrm{MBq}{ }^{90} \mathrm{Sr}\right)$ changes its direction during a Coulomb scattering process from a lead foil, by a kinematic effect and two additional precession effects. The kinematic effect is a geometrical effect, transferring the original longitudinal polarization to the transverse polarization in a Coulomb scattering between the electron and the lead nuclei, keeping its spin direction in a laboratory frame. In addition to this kinematic effect, Thomas precession must be considered, which is caused by a relativistic effect of electromagnetic interaction in a flat space-time. The tool of the present study is the gravitational geodetic precession. It is a general relativistic precession effect of a spinning object traveling in a warped space-time. The existence of this geodetic precession was confirmed in 2011 in the NASA satellite Gravity Probe B, as a precession of a gyroscope on an orbit around the Earth. We apply this phenomenon in the MTV-G experiment.

As shown in Figure 2, we utilize a Mott scattering to observe the transverse polarization of electrons, emitted from a radiation source and scattered by the first lead foil, where the precession effects are expected. In this Mott-analyser, the back scattering left-right asymmetry from the Mott scattering at a thin second lead foil is used as a measurement of the electron's transverse polarization. We used a MWDC (Multi-Wire Drift Chamber) to detect the electron tracks in the first experiment. This experiment is the application of the MTV detector, which is very sensitive to the tiny transverse polarization to search a T-Violation at TRIUMF-ISAC using polarized ${ }^{8} \mathrm{Li}[3]$. 
We performed a data taking of this experiment for two weeks at TRIUMF in 2011. An automatic source rotating device called "Auto-NS" was developed, which can change the source position at UP/DOWN configurations by a computer controlled system to be able to cancel the geometrical asymmetry of the detectors. We observed a clear parity violating transverse polarization in this measurement [2]. By comparing with the model calculations, we set a new constraint on the alphalambda plot at the shortest range as shown in Figure 3, after estimating the Thomas precession contribution. In order to reduce the remaining systematic effects arising from the detector's asymmetric acceptance, we upgraded the main detector from the MWDC to a CDC (cylindrical drift chamber). The new MTV-G experiment using the CDC was commissioned in 2012.

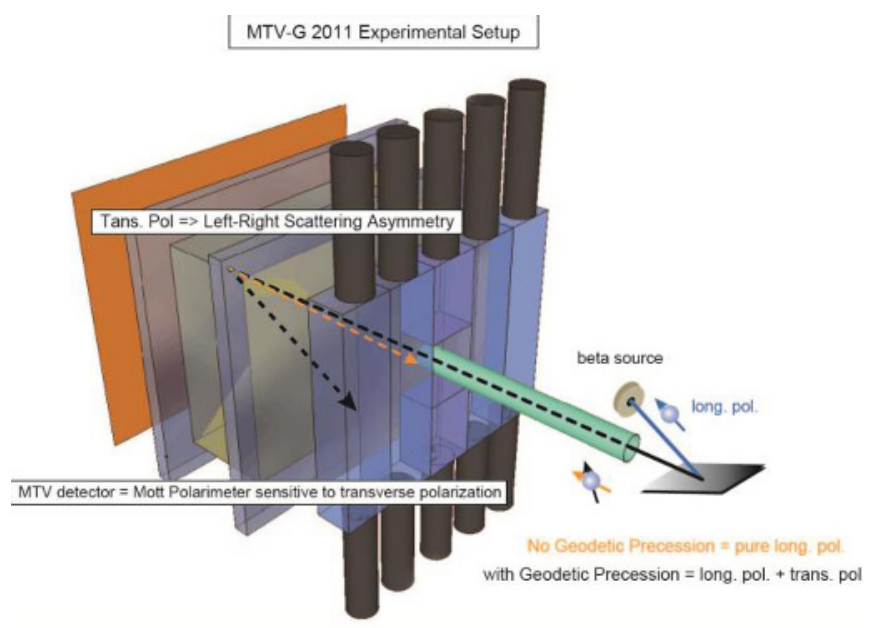

Figure 2. Experimental setup of the MTV-G experiment in 2011 using the MWDC.

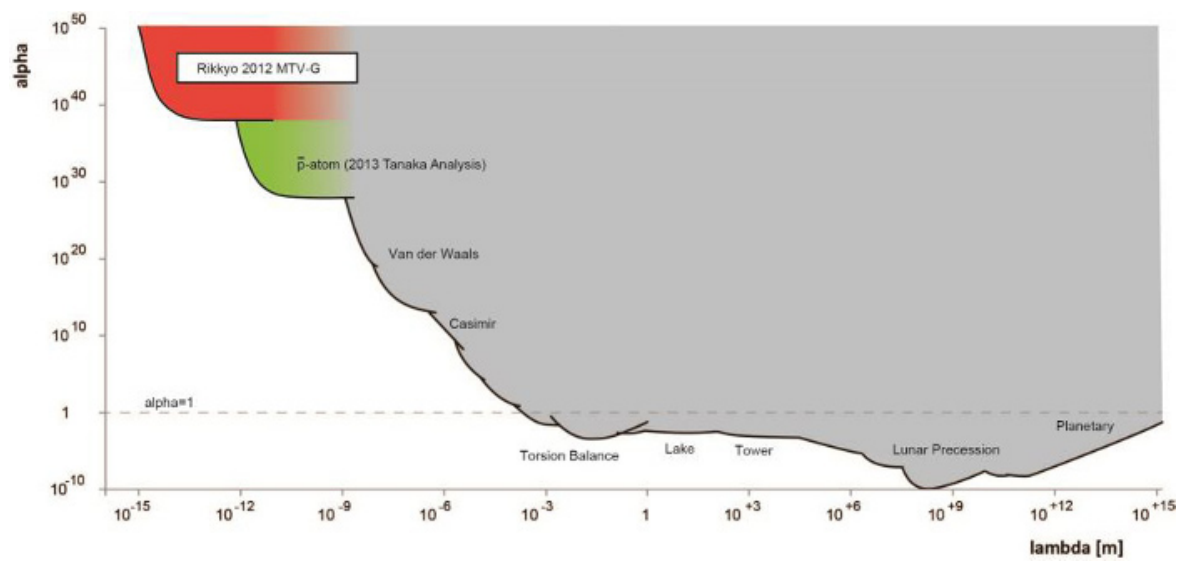

Figure 3. New constrains from the present studies in the alpha-lambda plot.

\section{Re-analysis of exotic atom}

In addition to the MTV-G experiment, we have also started to investigate existing data from spectroscopy of exotic atoms in scope of gravity. Based on the ADD model, the possible strong gravity should affect the energy levels, which can be tested in the transition wavelength. For example, we tried to extract the possible contribution from the new Yukawa term, by looking to an anti-protonic 
helium data [4]. In this experiment, the transition energy was measured with a precision of order $100 \mathrm{ppb}$ for the system shown in Figure 4, which is interpreted as the precision of alpha in our study.

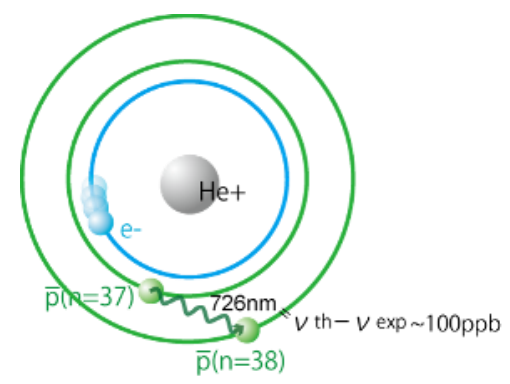

Figure 4. Atomic transition of anti-protonic helium [4].

We set a new alpha-lambda constraint by interpreting the present data using the non-Newtonian gravitational potential. The combined new constraints from the MTV-G experiment obtained in 2011 and from the re-analysis of this anti-protonic helium data are also plotted in the Figure 3.

\section{Future}

The first MTV-G experiment utilized the MWDC of the MTV experiment, which was not designed for this application. In order to reduce the remaining systematic effects, the CDC was designed and built, which was tested in 2012. We are ready to perform the final physics data taking of the MTV-G experiment in 2013 using the CDC setup, shown in Figure 5. In addition, the re-analysis of the existing exotic atom spectroscopy data will be finalized. The combined constrains in the alpha-lambda space at below pico-meter scale will be completed soon.

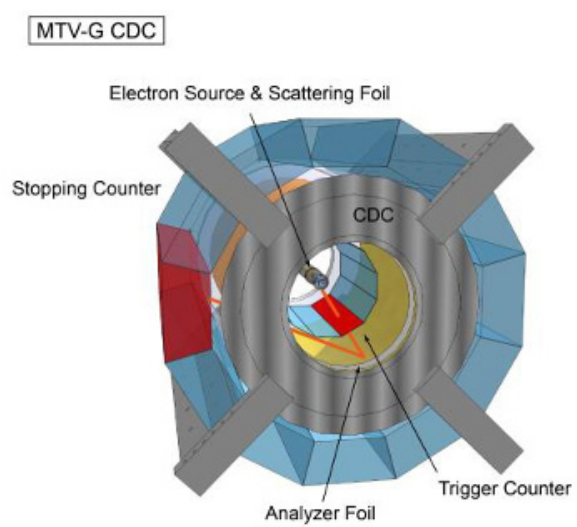

Figure 5. Experimental setup with CDC for the MTV-G experiment in 2013.

\section{References}

[1] N. Arkani-Hamed, S. Dimopoulo, G. Dvali, Phys. Lett. B429 (1998) 263.

[2] S. Tanaka et. al., J. Phys. CS453 (2013) 012018

[3] J. Murata et. al., J. Phys. CS312 (2011) 102011; Hyp. Int. 10.1007/s10751-013-0897-3 (2013)

[4] M. Hori et. al., Phys. Rev. Lett. 91 (2003) 123401-1 